\title{
Dose selection method for pharmacokinetic study in hemodialysis patients using a subpharmacological dose: oseltamivir as a model drug
}

Dong Ki Kim ${ }^{1 \dagger}$, Jay Wook Lee ${ }^{2 \dagger}$, Kwang-Hee Shin ${ }^{3}$, Sejoong Kim ${ }^{4}$, Kook-Hwan Oh' ${ }^{1}$ Myounghee Kim ${ }^{5}$ Kyung-Sang Yu ${ }^{6}$, Jung Pyo Lee ${ }^{7}$, Chun-Soo Lim7, Yon Su Kim ${ }^{1}$ and Kwon Wook Joo ${ }^{1,8^{*}}$

\begin{abstract}
Background: Dose selection is an important step in pharmacokinetic (PK) studies of hemodialysis patients. We propose a simulation-based dose-selection method for PK studies of hemodialysis patients using a subpharmacological dose of oseltamivir as a model drug.

Methods: The concentrations of oseltamivir and its active metabolite, oseltamivir carboxylate (OC), were measured by liquid chromatography-tandem mass spectrometry. To determine a low oseltamivir dose exhibiting PK linearity, a pilot low dose determination investigation $(n=4)$ was performed using a single administration dose-escalation study. After the dose was determined, a low dose study $(n=10)$ was performed, and the optimal dose required to reach the hypothetical target $O C$ exposure (area under the concentration-time curve [AUC] of 60,000 $\mathrm{ng} \cdot \mathrm{hr} / \mathrm{mL}$ ) was simulated using a nonparametric superposition method. Finally, observed PKs at the optimal dose were compared to the simulated PKs to verify PK predictability.

Results: In the pilot low dose determination study, $2.5 \mathrm{mg}$ of oseltamivir was determined to be the low dose. Subsequently, we performed a single-dose PK study with the low oseltamivir dose in an additional group of 10 hemodialysis patients. The predicted $\mathrm{AUC}_{\text {last }}$ of $\mathrm{OC}$ following continuous oseltamivir doses was simulated, and $35 \mathrm{mg}$ of oseltamivir corresponded to the hypothetical target $A \cup C_{\text {last }}$ of OC. The observed PK profiles of OC at a 35-mg oseltamivir dose and the simulated data based on the low dose study were in close alignment.

Conclusion: The results indicate that the proposed method provides a rational approach to determine the proper PK dose in hemodialysis patients.
\end{abstract}

Keywords: Hemodialysis, Pharmacokinetics, Drug, Dosage

\section{Background}

In addition to impaired renal function, hemodialysis patients may also possess altered pharmacokinetics (PKs) caused by hemodialysis itself. Hemodialysis is an important drug elimination rout, which is influenced by the characteristics of the drug, dialysis membrane and dose

\footnotetext{
* Correspondence: junephro@paran.com

${ }^{\dagger}$ Equal contributors

'Department of Internal Medicine, Seoul National University College of Medicine, Seoul, Korea

${ }^{8}$ Department of Internal Medicine, Seoul National University Hospital, 101 Daehak-ro, Jongro-gu, Seoul 110-744, Korea

Full list of author information is available at the end of the article
}

of dialysis [1]. In addition, hemodialysis may influence the metabolic clearance of a drug by affecting drugmetabolizing enzymes or transporters [2]. However, despite the difficulty of predicting PKs, which results from various interfering factors, previous PK studies have often excluded the hemodialysis patient population $[3,4]$. One reason for frequent exclusion is the uncertainty of selecting proper dosing for a PK study. In fact, improper dosing during PK studies can cause several problems. For example, administration of a potentially unsafe high dose during a PK study may lead to drug accumulation and adverse drug reactions, particularly in hemodialysis

\section{Biomed Central}


patients. Conversely, administering a dose that is too low may lead to unnecessary PK study repetition. Therefore, our objective was to develop a dose selection method for PK studies in hemodialysis patients using the simulationextrapolation of an optimal PK dose from the PK parameters of a subpharmacological dose.

In this study, we chose oseltamivir as a model drug to evaluate this proposed method because PKs of its active metabolite, oseltamivir carboxylate (OC), were dramatically altered, especially in patients undergoing hemodialysis [5]. Orally administered oseltamivir is rapidly converted into $\mathrm{OC}$ by first pass metabolism, which is minimally affected by liver function [6]. Consequently, approximately $80 \%$ of orally administered oseltamivir reaches the systemic circulation as $\mathrm{OC}$, and plasma OC concentrations exhibit minimal inter- and intra-subject variability [7]. The excretion of OC occurs exclusively via the kidneys, and therefore, $\mathrm{OC}$ exposure is substantially increased in patients with end-stage renal disease (ESRD) [7,8]. In addition, hemodialysis may also affect the PKs of oseltamivir because a significant fraction of $\mathrm{OC}$ is removed by hemodialysis [5,7]. Moreover, clinical concerns have arisen regarding oseltamivir use in patients with ESRD because of their high mortality from influenza, generally severe courses of influenza infection, and reduced response to vaccinations [9]. These PK and clinical characteristics of oseltamivir correspond to a drug category where the U.S. FDA recommends mandatory PK studies in patients with impaired renal function [10]. Therefore, we selected oseltamivir, even though this drug is already approved for mass marketing and its therapeutic dose PKs in ESRD patients are well known [5].

\section{Methods}

This study was conducted at the Clinical Trials Center and Dialysis Unit of Seoul National University Hospital (Seoul, Korea) from March 2011 to March 2012. The Institutional Review Board and the Korean Food and Drug Administration approved the study protocol. All procedures were performed in accordance with the recommendations of the Declaration of Helsinki. All subjects provided written informed consent and received prorated compensation after the study.

\section{Study subjects}

Eligibility criteria were an age $>20$ years and anuric nondiabetic ESRD undergoing intermittent hemodialysis. Exclusion criteria included current pregnancy or lactation, the prior use of an antiviral agent within the past three months, a history of allergic reaction to oseltamivir, alcohol and/or drug abuse, and participation in any other clinical study within two months prior to the present study. Additionally, subjects with gastrointestinal disease that could alter drug absorption were also excluded from the study. All other medications except anti-hypertensives were prohibited from three days prior to oseltamivir administration to the end of the study. As a safety precaution, subjects underwent a screening evaluation, including a medical history, vital signs, 12-lead electrocardiography, and clinical laboratory tests (complete blood count and serum chemistry profiles) that were performed within 4 weeks of oseltamivir administration. Subjects were fasted $8 \mathrm{hr}$ prior and $4 \mathrm{hr}$ following oseltamivir administration. During the admission period, subjects received and ate only the standard food and drink provided by the clinical trials center and were not allowed to consume fruit juice or any beverage containing alcohol or caffeine. All observed or self-reported adverse events that occurred over the study period were recorded. Blood pressure, pulse rate, and body temperature were measured at each sampling time.

\section{Study design}

\section{Step 1: pilot low dose determination study}

The dose-finding step was designed as an open-label, single sequence dose escalation, single-dosing study to determine the lowest oseltamivir dose detectable in plasma by liquid chromatography-tandem mass spectrometry (LCMS/MS) and showing dose-linearity with two higher oseltamivir doses. A total of four hemodialysis patients were enrolled in this pilot study. Considering the lower limit of quantification (LLOQ) for plasma oseltamivir concentrations $(0.2 \mathrm{ng} / \mathrm{mL})$ by LC-MS/MS as well as the oseltamivir PKs in patients with advanced renal dysfunction not on dialysis [7], a 2.5-mg oseltamivir dose was expected as the lowest detectable dose. The PK studies were performed using 3-week wash-out periods with dose doubling until the PK parameters at certain doses exhibited doselinearity with the PK parameters of two higher doses. All procedures were identical in all periods except for the dose administered. All patients were dialyzed for 4 hr using a high-flux membrane (Polyflux 170H; Gambro, Lund, Sweden; ultrafiltration coefficient, $70 \mathrm{~mL} /$ $\mathrm{hr} / \mathrm{Kg}$; single use). Blood flow was maintained at a constant rate of $250 \mathrm{~mL} / \mathrm{min}$, and the dialysate flow rate was also held constant at $500 \mathrm{~mL} / \mathrm{min}$.

\section{Step 2: low dose PK study and extrapolation of the appropriate $P K$ dose}

After determining the low oseltamivir dose during the pilot study, an additional set of 10 hemodialysis patients were evaluated in a single dose standard PK study using the low oseltamivir dose. Subsequently, the predicted area under the concentration-time curve (AUC) from drug administration to $72 \mathrm{hr}$ post-administration or to the last measurable time point ( $\mathrm{AUC}_{\text {last }}$ ) of $\mathrm{OC}$ following continuous doses up to $150 \mathrm{mg}$ was simulated from the low dose data using a nonparametric superposition method with 
the WinNolin ${ }^{\ominus}$ version 6.3 software (Pharsight Corporation, Mountain View, CA, USA). Based on the results of a previous oseltamivir PK study in hemodialysis patients [5], a hypothetical target $\mathrm{AUC}_{\text {last }}$ of $\mathrm{OC}$ was defined as $60,000 \mathrm{ng} \cdot \mathrm{hr} / \mathrm{mL}$, and the oseltamivir dose required to reach this target was considered to be the optimal PK dose.

\section{Step 3: method validation}

To validate the dose-selection method, the same participants were evaluated in a PK study using the extrapolated dose after a 3-week wash-out period. The observed PKs were compared to the simulated PKs based on the low dose study. The simulations for plasma oseltamivir, plasma $\mathrm{OC}$, and dialysate $\mathrm{OC}$ after the administration of the extrapolated oseltamivir dose were performed using a nonparametric superposition method with the WinNolin ${ }^{\circledR}$ version 6.3 software.

\section{PK assessment}

The study subjects received a solution containing oseltamivir dissolved in $20 \mathrm{~mL}$ of purified water followed by the administration of $150 \mathrm{~mL}$ of water immediately after the completion of hemodialysis. Blood samples were collected before oseltamivir administration and at 1, 1.5, 2, 2.5, 3, 6, 12, 24, 44 (dialysis start), 48 (dialysis end), and $72 \mathrm{hr}$ after oseltamivir administration through an indwelling catheter or direct venipuncture into pre-cooled fluoride tubes (BD, Franklin Lakes, NJ, USA). Plasma was separated from blood by centrifugation at $4^{\circ} \mathrm{C}$ within $2 \mathrm{hr}$ of collection. Dialysate was also collected hourly during the dialysis. All samples were stored at $-70^{\circ} \mathrm{C}$ until further use.

\section{Determination of plasma and dialysate oseltamivir and OC concentrations}

Oseltamivir, OC, and their trideuterated species used as internal standards were kindly provided by Hoffmann-La Roche (Basel, Switzerland). The quantification of plasma and dialysate oseltamivir and $\mathrm{OC}$ were performed using an Agilent 6460 triple quadrupole mass spectrometer (Palo Alto, CA, USA) coupled to an Agilent 1260 LC system (Palo Alto) using oseltamivir d3-carboxylate as an internal standard. The LLOQ were determined from standard curves of oseltamivir $(0.2-100 \mathrm{ng} / \mathrm{ml})$ and OC (2-500 ng/mL). The LLOQ was $0.2 \mathrm{ng} / \mathrm{ml}$ for oseltamivir and $2 \mathrm{ng} / \mathrm{mL}$ for OC. The coefficients of determination of the calibration curves were all $>0.99$. Standard curves were accurate $(93.7 \% \leq \%$ accuracy $\leq 101.2 \%)$ and precise $(0.775 \% \leq \%$ precision $\leq 6.924 \%)$ across a range of 0.5 to $80 \mathrm{ng} / \mathrm{mL}$ for oseltamivir and were accurate $(98.8 \% \leq \%$ accuracy $\leq 103.5 \%)$ and precise $(1.032 \% \leq \%$ precision $\leq 2.857 \%$ ) across a range of 5 to $400 \mathrm{ng} / \mathrm{mL}$ for $\mathrm{OC}$.

\section{PK and statistical analyses}

The PK parameters were calculated by a non-com partmental method using Phoenix ${ }^{\oplus}$ The $\mathrm{AUC}_{\text {last }}$ was computed using the linear trapezoidal approximation method. The maximum observed plasma concentration $\left(\mathrm{C}_{\max }\right)$ and time to reach $\mathrm{C}_{\max }\left(\mathrm{T}_{\max }\right)$ were directly determined from the individual concentration-time profiles. The oral plasma clearance $(\mathrm{CL} / \mathrm{F})$ was calculated as the dose/AUC $\mathrm{Ast}_{\text {last }}$ Intradialytic oseltamivir and OC clearance were calculated using the recovery method and the following equation: $C L_{H D}=R / A U C_{H D}$, where $C L_{H D}$ is the oseltamivir or OC clearance through hemodialysis, $\mathrm{R}$ is the amount of oseltamivir or OC recovered during the dialysis session, and $\mathrm{AUC}_{\mathrm{HD}}$ is the area under the serum concentration-time curve during hemodialysis.

The Kruskal-Wallis test was used to compare the dosenormalized $\mathrm{C}_{\max }$ and $\mathrm{AUC}_{\text {last }}$ between doses of oseltamivir. The dose proportionality in the dose-finding pilot study was tested using a power model analysis of logtransformed PK parameters versus the log-transformed dose. Dose proportionality was assumed if the slope was not statistically significantly different from unity and the 95\% confidence interval (CI) included 1.0 [11]. The extrapolated dose of oseltamivir was simulated using nonparametric superposition (WinNolin ${ }^{\circledR}$ version 6.3 software), which can be used to predict drug concentrations after multiple dosing at steady state, based on non-compartmental results of single dose data and not assuming any PK model [12].

Table 1 Demographic data for study subjects

\begin{tabular}{|c|c|c|}
\hline & $\begin{array}{c}\text { Low dose } \\
\text { determination } \\
\text { pilot study }(n=4)\end{array}$ & $\begin{array}{l}\text { Low dose study } \\
\text { and method } \\
\text { validation }(n=10)\end{array}$ \\
\hline Age (yr) & $56.8(14.8)$ & $52.4(14.2)$ \\
\hline Sex (male: female) & $2: 2$ & $6: 4$ \\
\hline Dry weight (Kg) & $50.3(13.4)$ & $52.9(12.1)$ \\
\hline Body mass index $\left(\mathrm{kg} / \mathrm{m}^{2}\right)$ & $20.6(5.0)$ & $20.1(3.0)$ \\
\hline \multicolumn{3}{|l|}{ Cause of ESRD (n) } \\
\hline Chronic glomerulonephritis & 2 & 4 \\
\hline Hypertension & 1 & 3 \\
\hline Unknown & 1 & 3 \\
\hline Time on hemodialysis & $3.3(0.8)$ & $4.1(1.6)$ \\
\hline Hemoglobin (g/dL) & $10.50(0.58)$ & $10.88(0.92)$ \\
\hline \multicolumn{3}{|l|}{ Dialysis efficiency } \\
\hline \multicolumn{3}{|l|}{ (at $44-48 \mathrm{hr}$ of study period) } \\
\hline Single-pool Kt/Nurea & $1.47(0.10)$ & $1.58(0.28)$ \\
\hline Urea reduction ration (\%) & $70.7(2.1)$ & $73.5(5.3)$ \\
\hline $\begin{array}{l}\text { Ultrafiltration rate } \\
\text { (L/session) }\end{array}$ & $2.60(0.13)$ & $2.10(0.76)$ \\
\hline
\end{tabular}

Data presented mean (standard deviation) or numbers. 

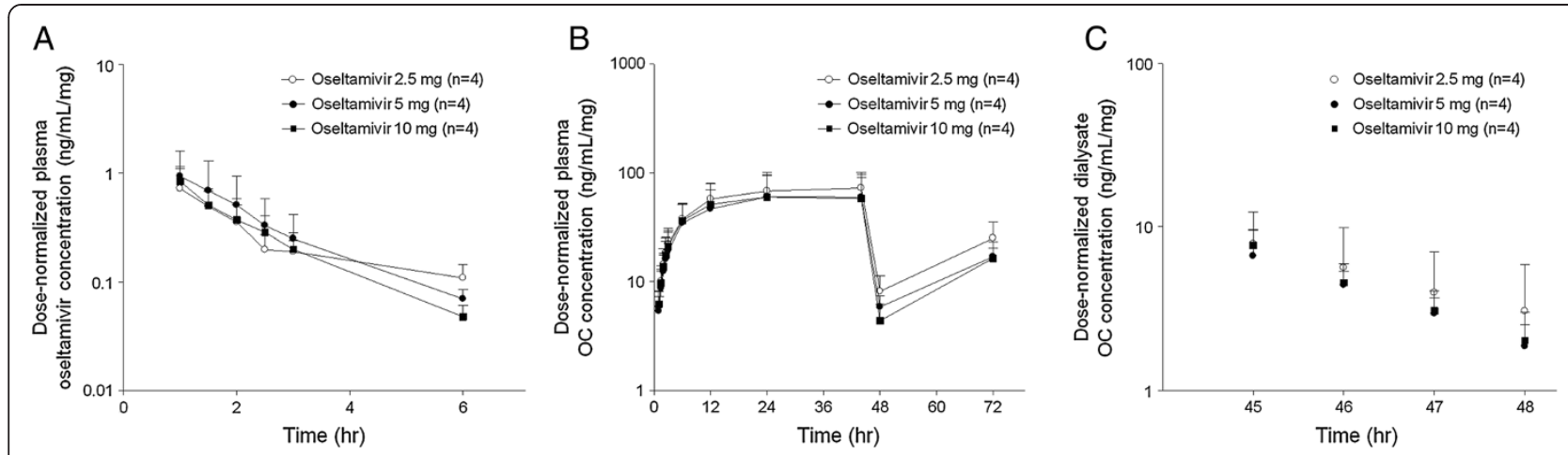

Figure 1 Semi-log plots of the dose-normalized mean plasma oseltamivir (A), plasma oseltamivir carboxylate (B), and dialysate oseltamivir carboxylate (C) concentrations following a single oral dose of $2.5(\circ), 5.0(\bullet)$, and $10.0 \mathrm{mg}(\square)$ of oseltamivir. Error bars represent the standard deviation. OC, oseltamivir carboxylate.

For PK data, summary statistics are presented as the means (SD). SPSS (version 12.0; SPSS Korea, Seoul, Korea) was used for the statistical analyses, and a $P$ value of less than 0.05 was considered statistically significant.

\section{Results}

\section{Subjects}

The demographic characteristics of the study participants are listed in Table 1. No subjects exhibited adverse events after oseltamivir administration in the dose-finding pilot study. However, two of the 10 participants who received a dose of $35 \mathrm{mg}$ of oseltamivir experienced nausea, which was mild in intensity. No serious adverse events occurred during the study period.

\section{Pharmacokinetics}

\section{Pilot low dose determination study}

Figure 1 shows a semi-log plot of the mean plasma oseltamivir, plasma $\mathrm{OC}$, and dialysate $\mathrm{OC}$ concentrations normalized to a 1-mg oseltamivir dose versus time post administration of a single oral oseltamivir dose of 2.5, 5.0, and $10.0 \mathrm{mg}$. Although oseltamivir could not be detected after $6 \mathrm{hr}$, the shapes of the oseltamivir and OC dose response curves were similar, and the curves were predominantly superimposable at the various doses. Similarly, the mean $\mathrm{AUC}_{\text {last }}$ values of oseltamivir and $\mathrm{OC}$, and the $\mathrm{AUC}_{\mathrm{HD}}$ of $\mathrm{OC}$ increased in a dose-dependent manner (Table 2). Additionally, no differences were detected between groups with respect to the dose-normalized $\mathrm{C}_{\max }$ and $\mathrm{AUC}_{\text {last }}$ using the Kruskal-Wallis test (Table 3). Furthermore, power model analysis displayed doseproportional increases in the $\mathrm{AUC}_{\text {last }}$ and $\mathrm{C}_{\max }$ of oseltamivir and $\mathrm{OC}$ within a dose range of 2.5 to $10 \mathrm{mg}$. The slope estimates were close to unity for the $\mathrm{AUC}_{\text {last }}$ and $\mathrm{C}_{\max }$ of oseltamivir and $\mathrm{OC}$ and $\mathrm{AUC}_{\mathrm{HD}}$ of OC. All the corresponding 95\% CIs included 1.0 (Table 4).

Together, the dose relationships of $\mathrm{AUC}_{\text {last }}$ and $\mathrm{C}_{\max }$ for oseltamivir and $\mathrm{OC}$ within the 2.5 to $10 \mathrm{mg}$ dose range did not deviate from dose proportionality. Accordingly, the subsequent PK study was performed with an oral dose of $2.5 \mathrm{mg}$ of oseltamivir.

Table 2 Pharmacokinetic parameters of oseltamivir and oseltamivir carboxylate following a single oral administration of $2.5,5$, and $10 \mathrm{mg}$ of oseltamivir

\begin{tabular}{|c|c|c|c|c|c|c|}
\hline \multirow[t]{2}{*}{ Parameter } & \multicolumn{3}{|c|}{ Oseltamivir } & \multicolumn{3}{|c|}{ Oseltamivir carboxylate } \\
\hline & $\begin{array}{l}\text { Oseltamivir } \\
2.5 \mathrm{mg}\end{array}$ & $\begin{array}{l}\text { Oseltamivir } \\
5 \mathrm{mg}\end{array}$ & $\begin{array}{l}\text { Oseltamivir } \\
10 \mathrm{mg}\end{array}$ & $\begin{array}{l}\text { Oseltamivir } \\
2.5 \mathrm{mg}\end{array}$ & $\begin{array}{l}\text { Oseltamivir } \\
5 \mathrm{mg}\end{array}$ & $\begin{array}{l}\text { Oseltamivir } \\
10 \mathrm{mg}\end{array}$ \\
\hline$C_{\max }(n g / m L)$ & $1.81(0.92)$ & $4.68(3.28)$ & $8.40(3.03)$ & 181.07 (96.38) & $410.35(206.54)$ & $599.23(360.64)$ \\
\hline $\mathrm{T}_{\max }(\mathrm{hr})$ & $1.00[1.00,1.55]$ & $1.06[1.00,1.13]$ & $1.00[1.00,1.55]$ & $44.11[24.00,44.25]$ & $43.99[24.0,44.05]$ & $24.01[24.00,43.68]$ \\
\hline$A \cup C_{\text {last }}(\mathrm{ng} \mathrm{h} / \mathrm{mL})$ & $3.24(0.81)$ & $9.60(6.83)$ & $15.47(5.48)$ & $7697.12(3930.52)$ & $12497.57(3537.45)$ & $25394.42(12912.41)$ \\
\hline$A \cup C_{H D}(\mathrm{ng} \mathrm{h} / \mathrm{mL})$ & NA & NA & NA & $288.52(119.78)$ & $458.82(226.88)$ & $784.87(205.39)$ \\
\hline $\mathrm{CL} / \mathrm{F}(\mathrm{L} / \mathrm{hr})$ & $554.41(37.86)$ & $558.27(252.14)$ & $645.73(161.19)$ & NA & NA & NA \\
\hline $\mathrm{CL}_{H D}(\mathrm{~mL} / \mathrm{hr})$ & NA & NA & NA & $5.14(1.87)$ & $6.41(3.43)$ & $6.92(1.30)$ \\
\hline Drug excreted by dialysis (\%) & NA & NA & NA & $0.06(0.04)$ & $0.05(0.02)$ & $0.04(0.01)$ \\
\hline
\end{tabular}

Data presented mean (SD) except $T_{\max }$ median [min, max]; $C_{\max }$, peak plasma concentration; $T_{\max }$ time to $C_{\max }$ AUC, area under the plasma concentration-time curve; $\mathrm{CL} / \mathrm{F}$, oral plasma clearance; $\mathrm{CL}_{\mathrm{HD}}$, dialysis clearance; $\mathrm{NA}$, not applicable; ${ }^{*}$ amount excreted of oseltamivir carboxylate / administered oseltamivir. 
Table 3 Dose-normalized $C_{\max }$ and $A U C_{\text {last }}$ following a single oral administration of 2.5, 5, and 10 mg of oseltamivir

\begin{tabular}{|c|c|c|c|c|c|c|c|c|}
\hline \multirow[t]{2}{*}{ Parameter } & \multicolumn{3}{|c|}{ Oseltamivir } & \multirow[t]{2}{*}{$P$-value ${ }^{*}$} & \multicolumn{3}{|c|}{ Oseltamivir carboxylate } & \multirow[t]{2}{*}{$P$-value* } \\
\hline & $\begin{array}{c}\text { Oseltamivir } \\
2.5 \mathrm{mg}(\mathrm{n}=4)\end{array}$ & $\begin{array}{l}\text { Oseltamivir } \\
5 \mathrm{mg}(\mathrm{n}=4)\end{array}$ & $\begin{array}{c}\text { Oseltamivir } \\
10 \mathrm{mg}(\mathrm{n}=4)\end{array}$ & & $\begin{array}{c}\text { Oseltamivir } \\
2.5 \mathrm{mg}(\mathrm{n}=4)\end{array}$ & $\begin{array}{l}\text { Oseltamivir } \\
5 \mathrm{mg}(\mathrm{n}=4)\end{array}$ & $\begin{array}{c}\text { Oseltamivir } \\
10 \mathrm{mg}(\mathrm{n}=4)\end{array}$ & \\
\hline $\mathrm{C}_{\max } /$ dose $(\mathrm{ng} / \mathrm{mL} / \mathrm{mg})$ & $0.72(0.37)$ & $0.94(0.66)$ & $0.84(0.30)$ & 0.981 & $72.43(38.55)$ & $82.07(41.31)$ & $59.92(36.06)$ & 0.926 \\
\hline $\mathrm{AUC}_{\text {last }} /$ dose $(\mathrm{ng} \cdot \mathrm{h} / \mathrm{mL} / \mathrm{mg})$ & $1.29(0.33)$ & $1.92(1.37)$ & $1.55(0.55)$ & 0.981 & $3078.9(1572.2)$ & 2499.5 (707.5) & $2539.4(1291.2)$ & 0.944 \\
\hline
\end{tabular}

Data presented mean (SD); $C_{\max }$ peak plasma concentration; AUC, area under the plasma concentration-time curve; ${ }^{*}$ The Kruskal-Wallis test.

\section{Low dose PK study and extrapolation of the appropriate PK dose}

After a low oseltamivir dose of $2.5 \mathrm{mg}$ was determined, we performed a single-dosing standard PK study following the administration of $2.5 \mathrm{mg}$ of oseltamivir with 10 additional hemodialysis patients. The mean $\mathrm{C}_{\max }$ of oseltamivir and OC were $1.63 \pm 0.87$ and $128.14 \pm 50.41 \mathrm{ng} /$ $\mathrm{mL}$, respectively, and the median $\mathrm{T}_{\max }$ of oseltamivir and OC were $1.00(0.98-1.00)$ and 33.95 (12.00-44.08) hr, respectively. The $\mathrm{AUC}_{\text {last }}$ values of oseltamivir and $\mathrm{OC}$ were $2.55 \pm 1.17$ and $5604.11 \pm 2097.22 \mathrm{ng} \cdot \mathrm{hr} / \mathrm{mL}$, respectively. The CL/F of oseltamivir was $618.84 \pm 424.92 \mathrm{~L} /$ $\mathrm{hr}$, and the $\mathrm{CL}_{\mathrm{HD}}$ of $\mathrm{OC}$ was $7.07 \pm 4.30 \mathrm{~mL} / \mathrm{hr}$. Subsequently, the predicted $\mathrm{AUC}_{\text {last }}$ values of $\mathrm{OC}$ following continuous doses were simulated from the low dose data using a nonparametric superposition method (Figure 2). The appropriate oral PK oseltamivir dose was determined to be $34.61 \mathrm{mg}$, which corresponded to the hypothetical target $\mathrm{AUC}_{\text {last }}$ of $60,000 \mathrm{ng} \cdot \mathrm{hr} / \mathrm{mL}$.

\section{Method validation}

The comparisons of the observed concentration-time profiles of plasma oseltamivir, plasma $\mathrm{OC}$, and dialysate $\mathrm{OC}$ at $35 \mathrm{mg}$ of oseltamivir (rounded up from $34.61 \mathrm{mg}$ ) and the simulated data based on the PK parameters at a dose of $2.5 \mathrm{mg}$ are shown in Figure 3. The simulated curves correspond closely to the measured values, indicating that low dose PK studies can be used to predict the proper PK dose in hemodialysis patients.

Table 4 Power model analysis for dose-concentration relationship

\begin{tabular}{lcc}
\hline Parameters & Estimate of $\boldsymbol{\beta}$ & $\mathbf{9 5 \%} \mathrm{Cl}$ \\
\hline Oseltamivir & & \\
$\mathrm{C}_{\max }(\mathrm{ng} / \mathrm{mL})$ & 1.187 & $0.556-1.818$ \\
$\mathrm{AUC}_{\text {last }}(\mathrm{ng} \mathrm{h} / \mathrm{mL})$ & 1.121 & $0.652-1.591$ \\
Oseltamivir carboxylate & & \\
$\mathrm{C}_{\max }(\mathrm{ng} / \mathrm{mL})$ & 0.889 & $0.232-1.547$ \\
$\mathrm{~A} \cup C_{\text {last }}(\mathrm{ng} \mathrm{h} / \mathrm{mL})$ & 0.897 & $0.374-1.421$ \\
$\mathrm{~A} \cup C_{\text {HD }}(\mathrm{ng} \mathrm{h} / \mathrm{mL})$ & 1.062 & $0.507-1.617$ \\
\hline
\end{tabular}

$\mathrm{C}_{\text {max }}$, peak plasma concentration; $\mathrm{Cl}$, confidence interval; $\mathrm{AUC}$, area under the plasma concentration-time curve.

\section{Discussion}

Although current guidelines recommend conducting a PK study for all investigational drugs that are likely be used in ESRD patients [10,13-15], only a small proportion of drugs have been evaluated for PKs in this population. For example, fewer than $30 \%$ of the new molecular entities approved by the U.S. FDA between 2003 and 2007 have had their PK profiles assessed in hemodialysis patients during drug development [4]. Moreover, PK studies evaluating drug efficacy and safety, which are usually conducted in phase 3 clinical trials, frequently exclude the hemodialysis patient population [3]. Accordingly, this paucity of PK studies may lead to non-optimized pharmacotherapies and medication-related problems, including drug dosing errors and inadequate efficacy [16]. In addition, the majority of the previously published PK data potentially underestimate the effects of dialysis on drug elimination because recently introduced dialyzers with high permeability and large surface areas were not examined [17-20]. Therefore, PK analyses for many drugs, some of which are already marketed, are necessary to optimize drug dosing in hemodialysis patients.

Individualized dosing recommendations that account for renal function can be derived from the estimation of PKs either through a simulation from detailed PK analyses in patients with varying degrees of renal function or a mixed-effect modeling approach using populationbased PK parameters obtained in large clinical studies [1]. However, given the impact of hemodialysis on drug PKs, PK profiles in this patient population cannot simply be extrapolated from the data of pre-dialysis patients with kidney disease [3]. Indeed, hemodialysis may not only eliminate a drug or its active metabolites but can also alter the PK through increased metabolic clearance through the dialysis-induced removal of endogenous inhibitors of metabolic enzymes [2]. Additionally, hemodialysis may decrease the intercompartmental drug clearance [19]. Therefore, standard PK studies in hemodialysis patients should be mandatory because of these dialysis-associated PK perturbations.

Dose selection in PK studies for hemodialysis patients may be an important practical step, and currently, U.S. FDA guidelines recommend using the same dose for all patients in renal impairment-PK studies regardless of the degree of renal function, because the peak concentration 


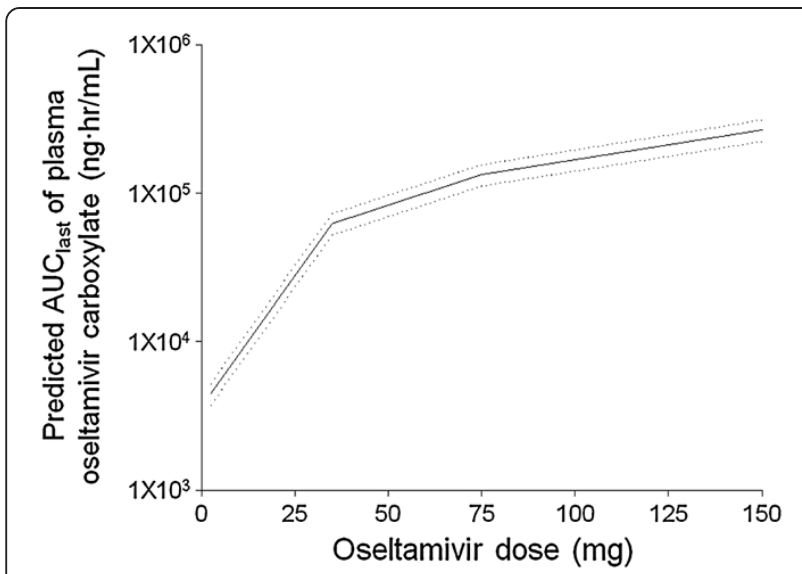

Figure 2 Predicted $A \cup C_{\text {last }}$ of plasma oseltamivir carboxylate following continuous doses simulated from the low dose data with a nonparametric superposition method. The solid and

dotted lines represent the fitted line and standard error, respectively. OC, oseltamivir carboxylate.

is not substantially affected by renal function [10]. However, in case of drugs with extensive renal excretion, such as oseltamivir, exposure to a drug or its active metabolites may be increased more than 10-fold in patients with severe renal impairment when an unadjusted dose is administered [7]. Moreover, approximately $70 \%$ of hemodialysis patients lose residual renal function within 1 year after the initiation of hemodialysis [21] and thus the majority of hemodialysis patients do not have residual renal function. Therefore, drug accumulation and adverse drug reactions during PK studies may be more prominent in these patients. In contrast, the selection of a sub-efficacious dose may cause unnecessary repetitious PK studies, thereby increasing study cost and duration. Therefore, a rational approach for dose selection that considers both safety and efficacy should precede the standard PK study, especially for drugs with renal excretion and low therapeutic range. In the present study, we employed oseltamivir as a model drug and demonstrated that PK profiles at therapeutic doses may be predicted using PK modeling and simulation based on low dose PK data. This finding suggests that low dose PK studies are useful in determining the dose used in detailed PK studies in hemodialysis patients. However, considerable debate exists regarding the prediction of the optimal PK dose from low dose studies because of the possibility of PK non-linearity between the doses. Indeed, the extrapolated prediction of the clinical PK parameters from a low dose study may not be accurate if a drug exhibits dose-dependent non-linear PKs [22]. To minimize the non-linearity concern, we performed a dose-escalation PK study in a small number of patients to select a low dose that exhibits linear PKs with two higher doses.

Although micro-dosing PK studies using less than $100 \mu \mathrm{g}$ of a drug can avoid toxicological effects [23], this approach requires expensive and specialized methodologies, such as positron emission tomography and accelerator mass spectrometry that require radioactive carbon-labeled drugs [24]. In addition, although many comparative studies for various drugs have shown PK linearity between the micro-dose and therapeutic dose [25-28], micro-dosing studies may not always accurately predict the PK parameters at higher therapeutic doses [29-31]. This non-linearity may arise from the non-linear disposition of a drug as well as the saturation of metabolic enzymes or drug transporters [32,33]. Therefore, the valid prediction of pharmacokinetics at therapeutic doses may be further complicated in micro-dosing studies in ESRD patients because impaired drug metabolism and dysfunction of drug transporters in patients with ESRD can alter non-renal clearance of drugs [34,35]. Thus, PK studies using non-radio-labeled drugs at low doses exhibiting linear pharmacokinetics with higher doses may present an alternative and simpler method to determine the PKs of a drug while avoiding unsafe higher doses.

The present study has several limitations that need to be addressed. First, the inter-compartmental clearance was not assessed. Second, the characteristics of OC rebound after dialysis were not fully assessed because no
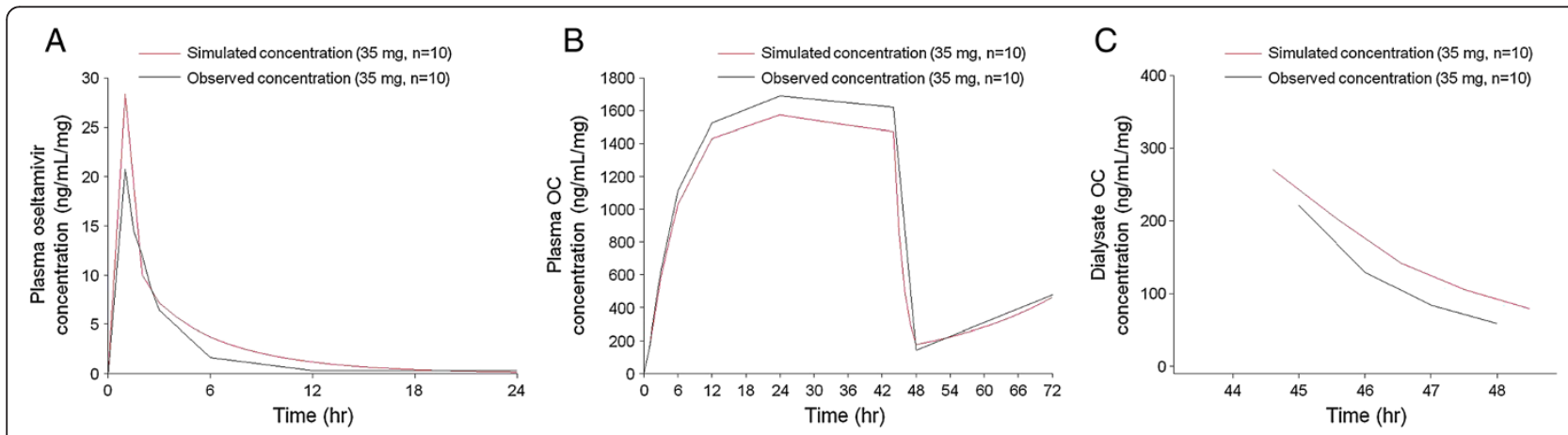

Figure 3 Simulated (red) and observed (black) concentration-time profiles of plasma oseltamivir (A), plasma oseltamivir carboxylate (B), and dialysate oseltamivir carboxylate (C) concentrations. OC, oseltamivir carboxylate. 
samples were collected after $48 \mathrm{hr}$. To evaluate the rebound quantitatively, further study should be conducted in HD patients using extended sampling time points.

\section{Conclusions}

In conclusion, the present study suggests that the proper PK drug dose for hemodialysis patients can be simulated from the PK parameters generated after the administration of a low dose at which adverse drug reactions are avoided.

\section{Abbreviations \\ AUC: Area under the concentration-time curve; CL: Clearance; $C_{\text {max }}$ : Maximum observed plasma concentration; ESRD: End-stage renal disease; LC-MS/MS: Liquid chromatography-tandem mass spectrometry; OC: Oseltamivir carboxylate; PK: Pharmacokinetic; $T_{\text {max }}$ : Time to reach maximum observed plasma concentration.}

\section{Competing interests}

The authors declare that they have no competing interests.

\section{Authors' contributions}

All authors contributed extensively to the work presented in this paper at all stage. DKK, JWL and KWJ conceived the design of this research and wrote the manuscript. KWJ supervised this study. SK, KHO, JPL, CSL, and YSK assembled input data. MK, KSY and KHS performed statistical analyses. JWL and DKK interpreted the data analyses. KWJ gave conceptual advice and commented on the manuscript. All authors read and approved the final manuscript.

\section{Acknowledgements}

This study was supported by a grant of the Korea Healthcare technology R\&D Project, Ministry for Health, Welfare \& Family Affairs, Republic of Korea (A070001). The biospecimens for this study were provided by the Seoul National University Hospital Human Biobank, a member of the National Biobank of Korea, which is supported by the Ministry of Health and Welfare. All samples derived from the National Biobank of Korea were obtained with informed consent under institutional review board-approved protocols.

\section{Author details}

'Department of Internal Medicine, Seoul National University College of Medicine, Seoul, Korea. ${ }^{2}$ Department of Internal Medicine, Chung-Ang University College of Medicine, Seoul, Korea. ${ }^{3}$ College of Pharmacy, Kyungpook National University, Daegu, Korea. ${ }^{4}$ Department of Internal Medicine, Seoul National University Bundang Hospital, Seongnam, Korea. ${ }^{5}$ Department of Dental Hygiene, College of Health Science, Eulji University, Seongnam, Korea. ${ }^{6}$ Department of Clinical Pharmacology and Therapeutics, Seoul National University College of Medicine, Seoul, Korea. ${ }^{7}$ Department of Internal Medicine, Seoul National University Boramae Medical Center, Seoul, Korea. ${ }^{8}$ Department of Internal Medicine, Seoul National University Hospital, 101 Daehak-ro, Jongro-gu, Seoul 110-744, Korea.

Received: 25 February 2013 Accepted: 11 March 2014

Published: 17 March 2014

\section{References}

1. Verbeeck RK, Musuamba FT: Pharmacokinetics and dosage adjustment in patients with renal dysfunction. Eur J Clin Pharmacol 2009, 65(8):757-773.

2. Nolin TD, Appiah K, Kendrick SA, Le P, McMonagle E, Himmelfarb J: Hemodialysis acutely improves hepatic CYP3A4 metabolic activity. J Am Soc Nephrol 2006, 17(9):2363-2367.

3. Edholm M, Gil Berglund E, Salmonson T: Regulatory aspects of pharmacokinetic profiling in special populations: a European perspective. Clin Pharmacokinet 2008, 47(11):693-701.

4. Zhang Y, Zhang L, Abraham S, Apparaju S, Wu TC, Strong JM, Xiao S, Atkinson AJ Jr, Thummel KE, Leeder JS, Lee C, Burckart GJ, Lesko LJ, Huang SM: Assessment of the impact of renal impairment on systemic exposure of new molecular entities: evaluation of recent new drug applications. Clin Pharmacol Ther 2009, 85(3):305-311.

5. Robson R, Buttimore A, Lynn K, Brewster M, Ward P: The pharmacokinetics and tolerability of oseltamivir suspension in patients on haemodialysis and continuous ambulatory peritoneal dialysis. Nephrol Dial Transplant 2006, 21(9):2556-2562.

6. Snell P, Dave N, Wilson K, Rowell L, Weil A, Galitz L, Robson R: Lack of effect of moderate hepatic impairment on the pharmacokinetics of oral oseltamivir and its metabolite oseltamivir carboxylate. $\mathrm{Br} J$ Clin Pharmacol 2005, 59(5):598-601.

7. He G, Massarella J, Ward P: Clinical pharmacokinetics of the prodrug oseltamivir and its active metabolite Ro 64-0802. Clin Pharmacokinet 1999, 37(6):471-484.

8. Massarella JW, He GZ, Dorr A, Nieforth K, Ward P, Brown A: The pharmacokinetics and tolerability of the oral neuraminidase inhibitor oseltamivir (Ro 64-0796/GS4104) in healthy adult and elderly volunteers. J Clin Pharmacol 2000, 40(8):836-843.

9. Marcelli D, Marelli C, Richards N: Influenza A(H1N1)v pandemic in the dialysis population: first wave results from an international survey. Nephrol Dial Transplant 2009, 24(12):3566-3572.

10. U.S. Department of Health and Human Services, Food and Drug Administration, Center for Drug Evaluation and Research: Guidance for Industry, Pharmacokinetics in patients with impaired renal function- Study design, data analysis, and impact on dosing and labeling; 2010. http://www. fda.gov/downloads/Drugs/Guidances/UCM204959.pdf.

11. Smith BP, Vandenhende FR, DeSante KA, Farid NA, Welch PA, Callaghan JT, Forgue ST: Confidence interval criteria for assessment of dose proportionality. Pharm Res 2000, 17(10):1278-1283.

12. Milo G, Donald P: Prediction of drug concentration on multiple dosing using the principle of superposition. In Pharmacokinetics. 2nd edition. New York: Marcel Dekker Inc; 1982:451-457.

13. European Medicines Agency, Committee for Medicinal Products for Human Use: Concept paper on the need for revision of the Note for guidance on the evaluation of the pharmacokinetics of medicinal products in patients with impaired renal function; 2012. http://www.ema.europa.eu/docs/en_GB/ document_library/Scientific_guideline/2012/06/WC500128218.pdf.

14. Zhang L, Xu N, Xiao S, Arya V, Zhao P, Lesko LJ, Huang SM: Regulatory perspectives on designing pharmacokinetic studies and optimizing labeling recommendations for patients with chronic kidney disease. J Clin Pharmacol 2012, 52(1 Suppl):79S-90S.

15. Huang SM, Temple R, Xiao S, Zhang L, Lesko LJ: When to conduct a renal impairment study during drug development: US Food and Drug Administration perspective. Clin Pharmacol Ther 2009, 86(5):475-479.

16. Manley HJ, Cannella CA, Bailie GR, St Peter WL: Medication-related problems in ambulatory hemodialysis patients: a pooled analysis. Am J Kidney Dis 2005, 46(4):669-680.

17. Matzke GR, Aronoff GR, Atkinson AJ Jr, Bennett WM, Decker BS, Eckardt KU, Golper T, Grabe DW, Kasiske B, Keller F, Kielstein JT, Mehta R, Mueller BA, Pasko DA, Schaefer F, Sica DA, Inker LA, Umans JG, Murray P: Drug dosing consideration in patients with acute and chronic kidney disease-a clinical update from Kidney Disease: Improving Global Outcomes (KDIGO). Kidney Int 2011, 80(11):1122-1137.

18. Dager WE: What are the important drug use errors in dialysis patients? Pharmacokinetic and pharmacodynamic principles. Semin Dial 2010, 23(5):466-469.

19. Atkinson AJ Jr, Umans JG: Pharmacokinetic studies in hemodialysis patients. Clin Pharmacol Ther 2009, 86(5):548-552.

20. Mueller BA, Smoyer WE: Challenges in developing evidence-based drug dosing guidelines for adults and children receiving renal replacement therapy. Clin Pharmacol Ther 2009, 86(5):479-482.

21. Moist LM, Port FK, Orzol SM, Young EW, Ostbye T, Wolfe RA, Hulbert-Shearon T, Jones CA, Bloembergen WE: Predictors of loss of residual renal function among new dialysis patients. J Am Soc Nephrol 2000, 11(3):556-564.

22. Benedetti MS, Whomsley R, Poggesi I, Cawello W, Mathy FX, Delporte ML, Papeleu P, Watelet JB: Drug metabolism and pharmacokinetics. Drug Metab Rev 2009, 41(3):344-390.

23. Garner RC, Lappin G: The phase 0 microdosing concept. Br J Clin Pharmacol 2006, 61(4):367-370.

24. Lappin G, Garner RC: Big physics, small doses: the use of AMS and PET in human microdosing of development drugs. Nat Rev Drug Discov 2003, 2(3):233-240. 
25. Lappin G, Kuhnz W, Jochemsen R, Kneer J, Chaudhary A, Oosterhuis B, Driffhout WJ, Rowland M, Garner RC: Use of microdosing to predict pharmacokinetics at the therapeutic dose: experience with 5 drugs. Clin Pharmacol Ther 2006, 80(3):203-215.

26. Lappin G, Shishikura Y, Jochemsen R, Weaver RJ, Gesson C, Brian Houston J, Oosterhuis B, Bjerrum OJ, Grynkiewicz G, Alder J, Rowland M, Garner C: Comparative pharmacokinetics between a microdose and therapeutic dose for clarithromycin, sumatriptan, propafenone, paracetamol (acetaminophen), and phenobarbital in human volunteers. Eur J Pharm Sci 2011, 43(3):141-150.

27. Yamazaki A, Kumagai Y, Yamane N, Tozuka Z, Sugiyama Y, Fujita T, Yokota S, Maeda M: Microdose study of a P-glycoprotein substrate, fexofenadine, using a non-radioisotope-labelled drug and LC/MS/MS. I Clin Pharm Ther 2010, 35(2):169-175.

28. Tozuka Z, Kusuhara H, Nozawa K, Hamabe Y, Ikushima I, Ikeda T, Sugiyama $Y$ : Microdose study of $14 C$-acetaminophen with accelerator mass spectrometry to examine pharmacokinetics of parent drug and metabolites in healthy subjects. Clin Pharmacol Ther 2010, 88(6):824-830.

29. Maeda K, Takano J, Ikeda Y, Fujita T, Oyama Y, Nozawa K, Kumagai Y, Sugiyama Y: Nonlinear pharmacokinetics of oral quinidine and verapamil in healthy subjects: a clinical microdosing study. Clin Pharmacol Ther 2011, 90(2):263-270.

30. Bertino JS Jr, Greenberg HE, Reed MD: American college of clinical pharmacology position statement on the use of microdosing in the drug development process. J Clin Pharmacol 2007, 47(4):418-422.

31. Beumer JH, Garner RC, Cohen MB, Galbraith S, Duncan GF, Griffin T, Beijnen $\mathrm{JH}$, Schellens $\mathrm{JH}$ : Human mass balance study of the novel anticancer agent ixabepilone using accelerator mass spectrometry. Invest New Drugs 2007, 25(4):327-334.

32. Sugiyama Y, Yamashita S: Impact of microdosing clinical study - why necessary and how useful? Adv Drug Deliv Rev 2011, 63(7):494-502.

33. Sparreboom A: Unexplored pharmacokinetic opportunities with microdosing in oncology. Clin Cancer Res 2007, 13(14):4033-4034

34. Reyes M, Benet LZ: Effects of uremic toxins on transport and metabolism of different biopharmaceutics drug disposition classification system xenobiotics. J Pharm Sci 2011, 100(9):3831-3842.

35. Momper JD, Venkataramanan R, Nolin TD: Nonrenal drug clearance in CKD: searching for the path less traveled. Adv Chronic Kidney Dis 2010, 17(5):384-391.

doi:10.1186/1471-2369-15-46

Cite this article as: Kim et al:: Dose selection method for pharmacokinetic study in hemodialysis patients using a subpharmacological dose: oseltamivir as a model drug. BMC Nephrology 2014 15:46.

\section{Submit your next manuscript to BioMed Central and take full advantage of:}

- Convenient online submission

- Thorough peer review

- No space constraints or color figure charges

- Immediate publication on acceptance

- Inclusion in PubMed, CAS, Scopus and Google Scholar

- Research which is freely available for redistribution 